\title{
Ion Yields for Some Salts in MALDI: Mechanism for the Gas-Phase Ion Formation from Preformed lons
}

\author{
Jeong Hee Moon, ${ }^{1}$ Young Sik Shin, ${ }^{2}$ Yong Jin Bae, ${ }^{2}$ Myung Soo Kim ${ }^{2}$ \\ ${ }^{1}$ Medical Proteomics Research Center, KRIBB, Daejeon 305-806, Korea \\ ${ }^{2}$ Department of Chemistry, Seoul National University, Seoul 151-742, Korea
}

\begin{abstract}
Preformed ion emission is the main assumption in one of the prevailing theories for peptide and protein ion formation in matrix-assisted laser desorption ionization (MALDI). Since salts are in preformed ion forms in the matrix-analyte mixture, they are ideal systems to study the characteristics of preformed ion emission. In this work, a reliable method to measure the ion yield (IY) in MALDI was developed and used for a solid salt benzyltriphenylphosphonium chloride and two room-temperature ionic liquids 1-butyl-3-methylimidazolium hexafluorophosphate and trihexyltetradecylphosphonium bis(2,4,4-trimethylpentyl)phosphinate. IY for the matrix (a-cyano-4-hydroxycinnamic acid, CHCA) was also measured. Taking 1 pmol salts in $25 \mathrm{nmol}$ CHCA as examples, IYs for three salts were similar, $(4-8) \times 10^{-4}$, and those for CHCA were $(0.8-1.2) \times 10^{-7}$. Even though IYs for the salts and CHCA remained virtually constant at low analyte concentration, they decreased as the salt concentrations increased. Two models, Model 1 and Model 2, were proposed to explain low IYs for the salts and the concentration dependences. Both models are based on the fact that the ion-pair formation equilibrium is highly shifted toward the neutral ion pair. In Model 1, the gas-phase analyte cations were proposed to originate from the same cations in the solid that were dielectrically screened from counter anions by matrix neutrals. In Model 2, preformed ions were assumed to be released from the solid sample in the form of neutral ion pairs and the anions in the ion pairs were assumed to be eliminated via reactions with matrix-derived cations.
\end{abstract}

Key words: Ion yield, Preformed ion, Ion formation mechanism, MALDI, Ion pair

\section{Introduction}

$\mathrm{E}$ ven though matrix-assisted laser desorption ionization (MALDI) [1-3] is widely used in the mass spectrometry of biological molecules, especially peptides and proteins, the ion formation process in this technique is not well understood. Two explanations for this process are competing

Electronic supplementary material The online version of this article (doi:10.1007/s13361-011-0278-6) contains supplementary material, which is available to authorized users.

Correspondence to: Myung Soo Kim; e-mail: myungsoo@snu.ac.kr at the moment, both of which are mainly focused on the formation of protonated peptides and proteins. One is the gasphase proton transfer model [4-8] and the other is the lucky survivor model [9-11]. In the gas-phase proton transfer model, a peptide is assumed to desorb in its neutral form $(\mathrm{P})$ and get protonated via gas-phase proton transfer with matrix-derived species such as $\mathrm{MH}^{+}$, namely, $\mathrm{P}+\mathrm{MH}^{+} \rightarrow \mathrm{PH}^{+}+\mathrm{M}$. Even though the details of the lucky survivor model have changed somewhat over the years, its main assumption remains the same. That is, a peptide is assumed to be present as a protonated form, or a preformed ion form, inside a solid matrix-analyte mixture and desorb in the form $\mathrm{PH}^{+}$or as clusters consisting of positive ions $\left(\mathrm{PH}^{+}\right)$, matrix neutrals, and negative counter-ions. 
In addition to the intact $\mathrm{PH}^{+}$ions, positively charged clusters are assumed to contribute to the analyte ion $\left(\mathrm{PH}^{+}\right)$signal through neutral loss after proton-transfer neutralization. The singly charged analyte ions surviving this process are called the lucky survivors of the neutralization.

Even though the ion yield (IY, number of ions formed : number of molecules in a sample) in MALDI must be apparently important for elucidating the ion formation mechanism, it has not received much attention presumably because of various experimental difficulties involved in its measurement. Standing et al. [12] counted the total number of analyte ions desorbed from a focused laser spot and estimated IY of $3 \times 10^{-4}$ for insulin in sinapic acid matrix. Mowry and Johnston [13] carried out MALDI for some alkylamines in various matrices and estimated the total number of co-desorbed neutrals by photoionization. IY thus estimated lied in the range of $10^{-5}-10^{-4}$ for the analytes. The matrix-to-analyte ratio of 1-10:1 adopted was much lower than in usual MALDI experiments. IYs for pure matrices were measured by two groups. Sundqvist et al. [14] measured the amount of desorbed ferulic acid deposited on a quartz crystal microbalance. IY estimated from the positive ion data in the reference is roughly $10^{-7}-10^{-6}$. Puretzky and Geohegan [15] measured the gas-phase density of desorbed 3-hydroxypicolinic acid by absorption spectroscopy. IY for this matrix becomes roughly $10^{-5}$ when estimated from the densities and spatial distributions for ions and neutrals they reported. In a recent monograph [1], Hillenkamp and Karas acknowledged that no accurate data were available for IY, but speculated that IYs for analytes and matrices would be around $10^{-3}-10^{-1}$ and $10^{-5}-10^{-3}$, respectively.

Even though IY data for analytes and matrices must contain information on the ion formation process and may also provide a clue for improving the performance of the MALDI technique, interpreting the experimental results for peptides can be troublesome because the occurrence of their protonation at some stage(s) of the overall process must be accommodated. For a salt added to a solid matrix, its positive and negative ions would be present as preformed ion forms. In this work, we developed a rather accurate method to measure IY, obtained the data for some salts, and attempted to seek information on the gas-phase ion formation mechanism from the data. We found that the possibility of the gas-phase proton transfer could not be completely ruled out even for the formation of gas-phase cations from salts.

\section{Experimental}

\section{Instrument}

Details of the homebuilt MALDI-TOF instrument and its operation were reported previously [16, 17]. Briefly, the instrument consists of an ion source with delayed extraction, a linear TOF analyzer, an ion gate, a second-stage analyzer equipped with a reflectron with linear-plus-quadratic (LPQ) potential inside, and a microchannel plate detector (MCP,
\#31849; Photonis USA, Sturbridge, MA, USA). The instrument was designed and tuned such that all the nondissociating ions coming out of the source would arrive at the detector. With 13 grids on the ion path, however, the theoretical transmission was 0.283 . $337 \mathrm{~nm}$ output from a nitrogen laser (MNL100; Lasertechnik Berlin, Berlin, Germany) focused by an $\mathrm{f}=100 \mathrm{~mm}$ lens was used for MALDI. Initially, the target and the extraction electrode in the source were kept at $20.0 \mathrm{kV}$ and the final electrode of the reflectron at 27.9. 200-400 ns (delay time) after the MALDI laser irradiation, $1.5 \mathrm{kV}$ was added to the target for ion extraction.

\section{Peaks Appearing in a MALDI Spectrum}

In a MALDI-TOF spectrum recorded by delayed extraction with an instrument equipped with a usual linear (L) reflectron, various matrix- and analyte-derived ions appear. They are the ions formed before the extraction pulse is applied and hence include prompt ions and those formed by the in-source decay (ISD) of some prompt ions. Unlike the L-reflectron, the LPQ-reflectron shows a good resolution for product ions formed between the ion source and the reflectron (post-source decay, PSD). Hence, not only the prompt ions and their ISD products but also the PSD products of such ions appear in a MALDI spectrum [18]. Identification of a PSD product ion can be further checked by recording a PSD spectrum, i.e. by activating the ion gate and hence selecting a precursor ion.

\section{Sample Preparation}

The matrix, $\alpha$-cyano-4-hydroxycinnamic acid (CHCA), the salts, benzyltriphenylphosphonium chloride (BTPP-Cl, mp> $300^{\circ} \mathrm{C}$ ), 1-butyl-3-methylimidazolium hexafluorophosphate $\left(\mathrm{BMI}-\mathrm{PF}_{6}, \mathrm{mp}=11^{\circ} \mathrm{C}\right)$, and trihexyltetradecylphosphonium bis(2,4,4-trimethylpentyl)phosphinate (THTDP-BTMPP, $\mathrm{mp}=-71^{\circ} \mathrm{C}$ ), and other chemicals were purchased from Sigma (St. Louis, MO, USA). Stock solutions of BTPP-Cl and BMI$\mathrm{PF}_{6}$ were prepared with 1:1 water/methanol mixture. The same solvent mixture was used to prepare CHCA stock solution used in the BTPP-Cl and BMI-PF 6 studies. In contrast, 1:1 water/ acetonitrile solution was used in the THTDP-BTMPP study (THTDP-BTMPP did not dissolve well in methanol solution). Each solution was successively diluted to get desired concentrations. Five microliters each of the matrix and salt solutions were mixed. One microliter of the mixture was loaded on a flat stainless steel target and vacuum-dried. The amount of CHCA in each sample loaded on the target was $25 \mathrm{nmol}$. The amount of each salt was $0.03-100$ pmol.

\section{Method}

\section{MCP Gain Calibration}

The method to calibrate the gain of the MCP detector was reported previously [19]. Briefly, the MCP gain was 
determined by integrating its output (current versus time) for a single ion pulse and averaging over 500 pulses. Two different gain curves (gain versus $\mathrm{m} / \mathrm{z}$ ) were used, one for the ions formed inside the source and the other outside (PSD). The isotopic patterns of strong peaks and overall MALDI spectral patterns were checked for signs of the detector saturation $[20,21]$. When suspicious, the experiment was repeated at lower MCP voltage. The MCP gain was used to calculate the number of each ion appearing in a spectrum.

\section{Measurement of Total Ion Intensity}

We measured the threshold laser pulse energy for MALDI of 1 pmol BTPP-Cl in $25 \mathrm{nmol} \mathrm{CHCA}$, which was $0.75 \mu \mathrm{J}$ per pulse. At a given pulse energy, we kept irradiating a spot with a focused laser, summed the spectra for every 20 shots, and displayed the shot-dependent changes in the intensities of some prominent analyte- and matrix-derived peaks. Irradiation stopped when hardly any ions appeared, which took 300-1000 shots depending on the analyte concentration and the laser pulse energy. The experiments were done at 2 , $3,4,6,8$, and 10 times the threshold pulse energy. Timeevolutions for the intensity of $\mathrm{BTPP}^{+}$and the sum of those for four CHCA-derived ions at $\mathrm{m} / \mathrm{z} 172,190,335$, and 379 obtained at 3, 6, and 10 times the threshold energy are shown in Figure 1. Surprisingly, the signal depletion occurred earlier at lower pulse energy. In such cases, however, the yellow color of CHCA remained, even though faintly. When such a sample was irradiated again with larger pulse energy than initially used, ion emission resumed even though with much lower intensity. With $6 \times$ threshold pulse energy, we could clearly see the burn mark on the sample formed by laser irradiation (Figure 2a). The area of the burn mark in the figure measured by a CCD camera (AM413T, Dinolite, The Netherlands) was around $0.0023 \mathrm{~mm}^{2}$. Using this area of the burn mark, we roughly estimated the ion yields (IY) and found that those at (4-10) $\times$ threshold energy were larger by around $30 \%$ than those at lower pulse energy. Larger IY at larger pulse energy might arise from more complete sample depletion. Alternatively, the effective area for ion emission might be larger at larger pulse energy. Hence, we decided to deplete samples from a much larger area than a focused laser spot. For this purpose, we depleted a focused laser spot, moved the target along the positive $\mathrm{x}$ direction with an xy-stage by an amount smaller than the laser spot size, depleted samples at this position, and moved the target again, etc. until a desired length along the $\mathrm{x}$-axis was covered. Then, we moved the target along the y-axis by a small amount, depleted samples, and moved the target along the negative $\mathrm{x}$ direction, etc. This enabled the collection of the ion intensity data over an extended area (Figure 2b). Through repeated measurements, we found that this extended area did not have to be larger than $85 \mu \mathrm{m} \times$ $250 \mu \mathrm{m}$ for reproducible results. We collected all the data at $6 \times$ threshold energy $(4.5 \mu \mathrm{J}$ per pulse) because the data collected at $(4-10) \times$ threshold energy were similar.

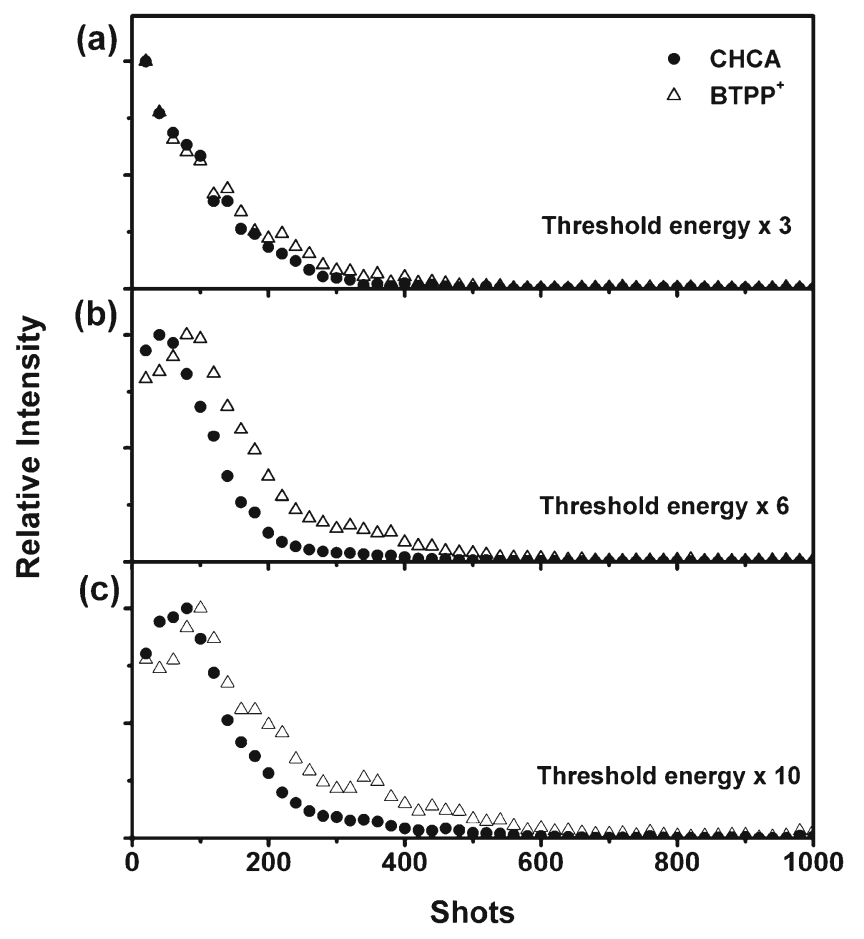

Figure 1. Time evolutions for the intensity of $\mathrm{BTPP}^{+}$and the sum of those for four CHCA-derived ions at $m / z 172,190$, 335, and 379 obtained at (a) 3, (b) 6, and (c) 10 times the threshold energy $(0.75 \mu \mathrm{J}$ per pulse) in MALDI of $1 \mathrm{pmol}$ $\mathrm{BTPP}-\mathrm{Cl}$ in $25 \mathrm{nmol} \mathrm{CHCA}$. Ion intensities were measured from the spectra accumulated for every 20 shots and normalized to the maximum in each time-evolution pattern. $337 \mathrm{~nm}$ laser was focused by an $\mathrm{f}=100 \mathrm{~mm}$ lens

\section{Sample Homogeneity}

It is well known that samples prepared for MALDI are generally inhomogeneous. In fact, it is a common practice in MALDI to seek sweet spots, i.e., spots generating good MALDI signals, using a microscope. In the present case, we wanted to measure the number of ions emitted from an extended area-collecting data from an entire sample was hopelessly time-consuming-and convert it to the total number expected from the sample by multiplying the (sample area/extended area) ratio. Then, the sample homogeneity became one of the prerequisites. When the usual airdried droplet method was used for CHCA, the sample looked quite inhomogeneous, with most of the CHCA crystallites forming near the edge of the dried sample. We attempted to estimate the ion intensities from air-dried samples by performing measurements for extended areas located at various positions and making a weighted sum. Even one such measurement took days.

With the vacuum-dried droplet method, the dried sample looked quite homogeneous. Actual measurement showed that the ion intensity at the center of the sample was lower than that at the edge by $50 \%$. Through many measurements, we found that representative and reproducible results could be obtained by collecting data from an extended area located 
(a)

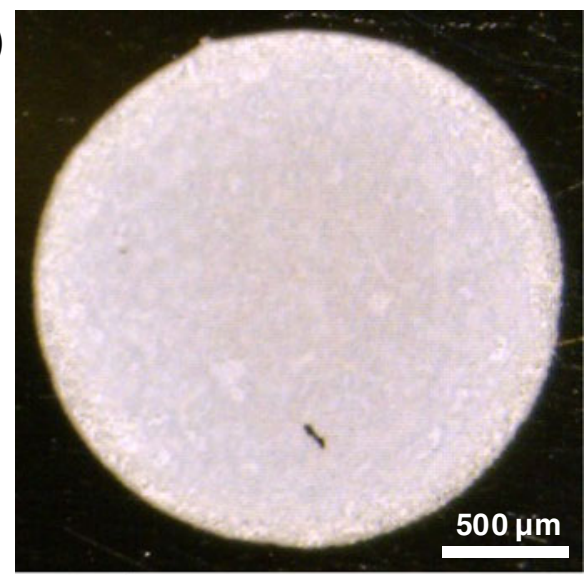

(b)

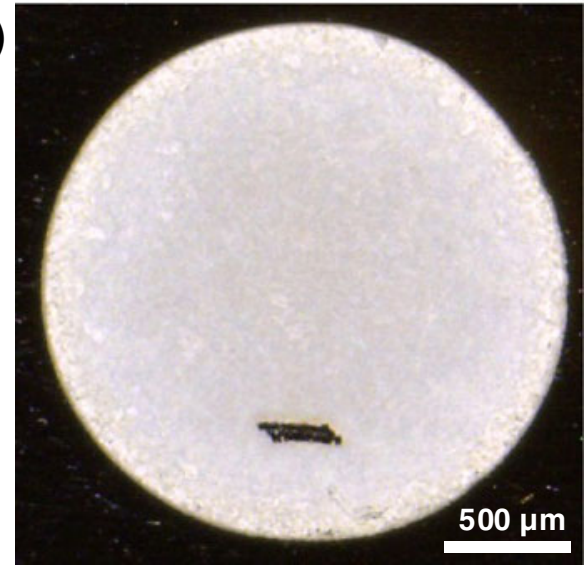

Figure 2. (a) A burn mark generated on a sample by repeated irradiation of a focused $337 \mathrm{~nm}$ MALDI laser until ion signals disappeared. $4.5 \mu \mathrm{J}$ per pulse of laser energy was used. The area of the burn mark measured by a CCD camera was around $0.0023 \mathrm{~mm}^{2}$. (b) A burn mark formed on an extended area $(85 \mu \mathrm{m} \times 250 \mu \mathrm{m})$ of a sample

at the half way between the center and the edge. Judging from a limited number of measurements, IYs for air- and vacuum-dried samples were similar.

\section{Correction for Ion Survival}

Some prompt ions formed by MALDI undergo dissociation inside (ISD) and outside (PSD) the source. In our previous dissociation kinetic studies [18] for peptide ions generated by MALDI, we developed a method to estimate the probability for a precursor ion coming out of the source to survive at the detector $\left(\mathrm{S}_{\text {post }}\right)$. Taking into account the flight times of a precursor ion through various regions of the instrument, the actual extent of post-source dissociation was estimated to be larger than that measured in the PSD spectrum by a factor of 6 (a factor 9 for the instrument used in this work [17]). We also estimated the probability $\left(\mathrm{S}_{\mathrm{in}}\right)$ for a prompt ion to survive at the source exit by utilizing the intensities of ISD product ions and the PSD yield of each ISD product ion. Then, the overall survival probability of a prompt precursor ion at the detector was taken as $\mathrm{S}_{\mathrm{in}} \times \mathrm{S}_{\text {post }}$.
The method to correct for ion dissociation needed in this work is a little different because one wants to calculate the total number of ions originating from the analyte, or from the matrix, whether an ion is in the form of a precursor ion, ISD and PSD products, or PSD of an ISD product. The easiest way is to identify a PSD peak in a MALDI spectrum and multiply its intensity by 9 to correct for the ion disappearance in regions like inside the reflectron. The extents of dissociation for the analyteand matrix-derived prompt ions studied in this work were quite low. Hence, the corrections made for the ion survival were rather insignificant.

\section{Determination of IY}

To summarize, the number of ions in each peak of a MALDI spectrum accumulated over an extended area was calculated by taking into account the ion survival, transmission $(28.3 \%$ transmission through the instrument and $62 \%$ entrance to microchannels in MCP), and the MCP gain. The number of ions originating from an analyte were summed including those from higher mass isotopes and multiplied by the (sample area/extended area) ratio to determine the total number of ions expected from the whole sample. Finally, it was divided by the number of the analyte molecules loaded on the target to get IY. The same method was used to determine IY for the matrix.

\section{Results and Discussion}

\section{Spectra}

The MALDI spectra for BTPP-Cl, BMI-PF 6 , and THTDPBTMPP obtained with 1 pmol each in $25 \mathrm{nmol}$ CHCA are shown in Figure 3. Most of the peaks appearing in the three spectra are CHCA-derived and their patterns are hardly affected by the analytes added. $\mathrm{P}\left(\mathrm{C}_{6} \mathrm{H}_{5}\right)_{3}{ }^{+}(\mathrm{m} / z$ 262), $\mathrm{P}$ $\left(\mathrm{C}_{6} \mathrm{H}_{5}\right)_{2}{ }^{+}\left(\mathrm{m} / \mathrm{z}\right.$ 185), and $\mathrm{C}_{7} \mathrm{H}_{7}{ }^{+}(\mathrm{m} / \mathrm{z}$ 91) appeared in the MALDI-PSD spectrum (not shown) of $\mathrm{BTPP}^{+}$. All of these were formed by ISD also, as marked in Figure 3a. Peaks at $m / z 185$ and 91 seemed to be heavily contaminated by CHCA-derived ions. In addition, the very weak PSD peak for $m / z 262$ could be identified. However, the other PSD peaks were too weak to identify. Hence, in measuring the number of ions derived from BTPP-Cl, we summed those for $\operatorname{BTPP}^{+}(m / z$ 353) and ISD and PSD peaks for $m / z 262$. Adding ISD and PSD intensities to that of the main cation $\left(\mathrm{BTPP}^{+}\right.$in this case) resulted in very small correction for all the salts studied and for CHCA also. In the case of BMI-PF ${ }_{6}$, the intensities of the ISD and PSD peaks for $\mathrm{m} / \mathrm{z} 83$ $\left(\mathrm{C}_{4} \mathrm{H}_{7} \mathrm{~N}_{2}{ }^{+}\right)$were added to that of $\mathrm{BMI}^{+}$. In contrast, more than 30 product ion peaks appeared in the PSD spectrum of THTDP $^{+}$with the abundances in the range of 0.00010.0005 relative to the precursor ion intensity. Even though the intensities of the ISD for $m / z 455$ and the 


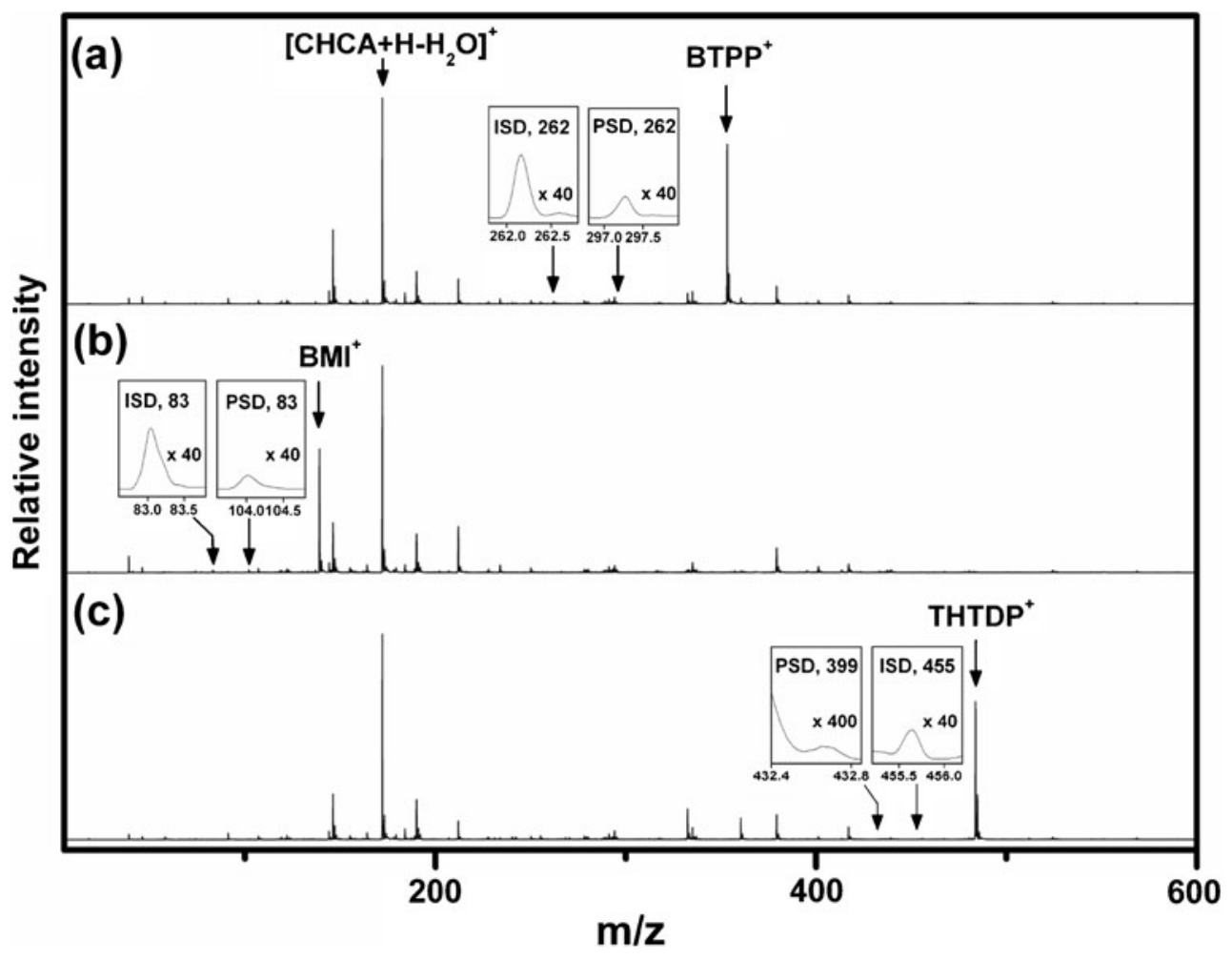

Figure 3. The MALDI spectra for (a) BTPP-Cl, (b) BMI-PF , and (c) THTDP-BTMPP obtained with 1 pmol of an analyte in $25 \mathrm{nmol} \mathrm{CHCA}$. Each spectrum was normalized to the intensity of [CHCA $\left.+\mathrm{H}-\mathrm{H}_{2} \mathrm{O}\right]^{+}(\mathrm{m} / z$ 172). Most of the peaks appearing in the three spectra are CHCA-derived. Some ISD and PSD product ion peaks for each analyte are marked

PSD for $m / z 399$ were added to that of $\mathrm{THTDP}^{+}$, the correction was insignificant.

\section{IY in MALDI of BTPP-Cl}

CHCA-MALDI experiments for BTPP-Cl were done for samples containing $25 \mathrm{nmol}$ of CHCA and 0.1-30 pmol of the analyte. There was a tiny CHCA-derived peak at the position of $\mathrm{BTPP}^{+}(\mathrm{m} / \mathrm{z} 353)$. Hence, determining the number of $\mathrm{BTPP}^{+}$became unreliable when the amount of the analyte was less than 0.1 pmol. Also, we felt suspicious of the MCP saturation when the amount of the analyte was somewhat larger than $30 \mathrm{pmol}$. As described in a previous section, we measured the number of the analyte-derived ions from an extended area on a sample, converted it to the number expected from the whole sample, and divided it by the number of molecules loaded on the target to get IY. Similar data for CHCA were obtained from the same spectra. At each analyte concentration, 4-7 duplicate measurements were made for 3-5 different samples. The numbers of ions from the whole samples and IY data are listed in Table 1.

IY for CHCA determined in this work, $0.2-1.2 \times 10^{-7}$ is somewhat smaller than $10^{-7}-10^{-6}$ for ferulic acid estimated from the data reported by Sundqvist et al. [14]. Our results are much smaller than $10^{-5}$ estimated from the Puretzky and Geohegan's data [15] and even much smaller than $10^{-5}-10^{-3}$ speculated by Hillenkamp and Karas [1].
Intuitively, $\mathrm{BTPP}^{+}$, which is in the form of a preformed ion in the solid matrix-analyte mixture, might be readily released to the gas phase as a free ion once the matrix neutrals and counter-ions surrounding it are easily detached during the laser-induced ablation. In this regard, it was rather surprising to find that only a tiny fraction of $\mathrm{BTPP}^{+}$, less than $10^{-3}$, appeared as gas-phase ions. It is to be noted that IY for the analyte determined in this work is rather close to IY for insulin measured by Standing et al. [12] and IYs for alkylamines measured by Mowry and Johnston [13]. However, we are not in a position to compare their results with ours because $\mathrm{BTPP}^{+}$is apparently in a preformed ion form in the solid sample while the ion formation mechanism for insulin and alkylamines, which requires protonation, is not definitely known. We would like to add that IY

Table 1. The Total Numbers of BTPP-Cl- and CHCA-derived Positive Ions and their IYs in CHCA-MALDI of BTPP-Cl (Further Abbreviated as B-C) Measured as a Function of the Analyte Amount in 25 nmol CHCA

\begin{tabular}{lccccc}
\hline B-C, pmol & \multicolumn{2}{c}{ Ions from sample } & & \multicolumn{2}{c}{ IY } \\
\cline { 2 - 3 } \cline { 5 - 6 } & B-C, $10^{8}$ & CHCA, 10 & & B-C, $10^{-4}$ & CHCA, 10 \\
\hline 0.1 & $0.52 \pm 0.03$ & $1.8 \pm 0.2$ & & $8.6 \pm 0.5$ & $1.2 \pm 0.1$ \\
0.3 & $1.5 \pm 0.2$ & $2.1 \pm 0.4$ & & $8.2 \pm 1.1$ & $1.4 \pm 0.3$ \\
1 & $4.5 \pm 0.8$ & $1.8 \pm 0.2$ & & $7.5 \pm 1.4$ & $1.2 \pm 0.2$ \\
3 & $11 \pm 2$ & $1.4 \pm 0.2$ & & $6.3 \pm 0.8$ & $0.97 \pm 0.15$ \\
10 & $23 \pm 1$ & $0.81 \pm 0.11$ & & $3.8 \pm 0.2$ & $0.53 \pm 0.08$ \\
30 & $28 \pm 4$ & $0.29 \pm 0.11$ & & $1.6 \pm 0.3$ & $0.19 \pm 0.07$ \\
\hline
\end{tabular}


measured in this work for the preformed $\mathrm{BTPP}^{+}$is certainly smaller than $10^{-3}-10^{-1}$ speculated for peptides and proteins by Hillenkamp and Karas [1].

In the apparatus, several apertures were installed on the ion path, which might reduce ion transmission. We made our best effort [17] to assure full transmission of ions coming out of the source to the detector by adjusting instrumental conditions such as deflector potentials, inner diameters of apertures, etc. We cannot rule out the possibility that some ions with dynamical properties quite different from the averages may have been lost. Even though the systematic errors involved are thought to be rather small, we regard the IY values determined in this work as the lower limits.

\section{Cluster Ion Formation}

In the lucky survivor model [9-11], Karas et al. assumed that clusters consisting of preformed cations such as protonated peptides, matrix neutrals, and counter anions were released into the gas phase upon ablation, and that positive analyte ions were formed by break-up of positively charged clusters. Then, in MALDI of BTPP-Cl, cluster ions such as $[\mathrm{BTPP}]_{\mathrm{n}}[\mathrm{X}]_{\mathrm{m}}$ with $\mathrm{n}>\mathrm{m}$, where $\mathrm{X}$ can be any anion in the solid sample such as $\mathrm{Cl}^{-}$and $[\mathrm{CHCA}-\mathrm{H}]^{-}$, may contribute to the formation of $\mathrm{BTPP}^{+}$. Let us take $[\mathrm{BTPP}]_{2}{ }^{2}$ ${ }^{+}[\mathrm{Cl}]^{-}$as an example. Detachment of the ion pair BTPP-Cl from this cation would release $\mathrm{BTPP}^{+}$into the gas phase. If the reaction is not too rapid, the cluster ion may appear as an intact ion in the MALDI spectrum or show PSD peaks for the corresponding reaction in our MALDI and PSD spectra. We searched for such peaks in the MALDI spectrum of BTPP-Cl. We also attempted a similar search by setting the ion gate to transmit $[\mathrm{BTPP}]_{2}{ }^{2+} \mathrm{Cl}^{-}$, even though it was absent in the MALDI spectrum, and recording its PSD spectrum. The search was made for some combinations of $\mathrm{n}$ and $\mathrm{m}$ up to $n=4$ and for various $X$. However, the intensities of ion peaks observed at the positions expected for all the conceivable PSD products were tiny, only at the background noise level.

The failure to observe cluster ions may simply mean that such ions are not efficiently formed by MALDI. Alternatively, even though they are abundant in the selvedge or in the early plume, they may have recombined with anions and got neutralized. In both cases, cluster ions are not important intermediates in the formation of gas phase $\mathrm{BTPP}^{+}$. However, the fact that cluster ions were undetectable does not rule out the cluster ion mechanism because there is a possibility that these ions, which were abundant in the selvedge or in the early plume, may have lost neutrals very rapidly and formed $\mathrm{BTPP}^{+}$. Since the time scale for such reactions must be shorter (say, less than $100 \mathrm{~ns}$ ) than can be resolved by usual MALDI-TOF instruments with delayed extraction, the cluster ion mechanism becomes a matter of academic curiosity that cannot be proven at the current stage of technology-cluster ions were difficult to detect with the continuous extraction mode either. Hence, we will disregard the cluster ion mechanism from now on.

In an early version of the lucky survivor model [9], electrons generated in the MALDI process, especially the photoelectrons from the metal or the metal-matrix interface [22-24], were proposed to contribute to the neutralization of positive analyte ions. In this regard, we measured IYs for two samples loaded on an anchor target coated with a hydrophobic organic layer, one on the bare metal surface and the other on the organic layer. IYs thus obtained were similar, eliminating the neutralization by electrons as an explanation for small IYs observed in this work.

\section{IYs for Room-Temperature Ionic Liquids}

A simple-minded explanation for the small IY for BTPP-Cl is that a neutral ion pair of $\mathrm{BTPP}^{+}$and a counter-anion is bound by a strong Coulombic force and is not easily detached during ablation. For a neutralization reaction, $\mathrm{A}^{+}$ $+\mathrm{B}^{-} \rightarrow \mathrm{AB}$, the change in Gibbs free energy would be dominated by the change in the Coulombic potential $\left(V_{\mathrm{c}}\right)$, resulting in the following approximation for the equilibrium constant, $K_{\text {neutral }} \approx \exp \left(-V_{\mathrm{c}} / \mathrm{RT}\right)$. Let us consider an ion pair at $1000 \mathrm{~K}$ consisting of two unit charges of opposite signs. With the inter-charge distances of 5 and $6 \AA$, the equilibrium constants become $3 \times 10^{14}$ and $1 \times 10^{12}$, respectively. This simple calculation demonstrates that $K_{\text {neutral }}$, which is very large, decreases rapidly as the Coulombic force decreases with the increase in the inter-charge distance. Hoping to observe larger IYs for salts, we performed the experiments for two room-temperature ionic liquids [25], i.e., BMI-PF $_{6}\left(\mathrm{mp}=11^{\circ} \mathrm{C}\right)$ and THTDP-BTMPP $\left(\mathrm{mp}=-71^{\circ} \mathrm{C}\right)$, melting point might be a rough measure of the Coulombic force. Before presenting the results, we would like to mention that evidence for the formation of cluster ions was not found for the two room-temperature ionic liquids either. The numbers of the analyte- and matrix-derived cations and IYs measured for BMI-PF $_{6}$ and THTDP-BTMPP are listed in Tables 2 and 3, respectively. Taking $1 \mathrm{pmol}$ of analytes as examples, IYs for BTPP-Cl, BMI-PF 6 , and THTDP-BTMPP are $(7.5 \pm 1.4) \times 10$ ${ }^{-4},(3.5 \pm 0.3) \times 10^{-4}$, and $(6.2 \pm 0.9) \times 10^{-4}$, respectively. IYs for the three salts were similar. In particular, IY did not increase as

Table 2. The Total Numbers of $\mathrm{BMI}_{-} \mathrm{PF}_{6}$ - and $\mathrm{CHCA}$-derived Positive Ions and their IYs in CHCA-MALDI of BMI-PF $_{6}$ (Further Abbreviated as B-P) Measured as a Function of the Analyte Amount in $25 \mathrm{nmol}$ CHCA

\begin{tabular}{lcclcc}
\hline B-P, pmol & \multicolumn{2}{c}{ Ions from sample } & & \multicolumn{2}{c}{ IY } \\
\cline { 2 - 3 } \cline { 5 - 6 } & B-P, $10^{8}$ & CHCA, $10^{9}$ & & B-P, $10^{-4}$ & CHCA, $10^{-8}$ \\
\hline 0.03 & $0.15 \pm 0.06$ & $1.4 \pm 0.1$ & & $8.5 \pm 3.4$ & $8.9 \pm 0.7$ \\
0.1 & $0.52 \pm 0.09$ & $1.5 \pm 0.4$ & & $8.7 \pm 1.5$ & $9.7 \pm 2.3$ \\
0.3 & $0.71 \pm 0.14$ & $1.4 \pm 0.2$ & & $3.9 \pm 0.8$ & $9.1 \pm 1.1$ \\
1 & $2.1 \pm 0.2$ & $1.2 \pm 0.3$ & & $3.5 \pm 0.3$ & $8.0 \pm 2.1$ \\
3 & $6.0 \pm 0.4$ & $1.0 \pm 0.2$ & & $3.3 \pm 0.2$ & $6.6 \pm 1.6$ \\
10 & $9.8 \pm 1.1$ & $0.55 \pm 0.07$ & & $1.6 \pm 0.2$ & $3.6 \pm 0.5$ \\
30 & $22 \pm 4$ & $0.42 \pm 0.13$ & & $1.2 \pm 0.2$ & $2.8 \pm 0.9$ \\
\hline
\end{tabular}


Table 3. The Total Numbers of THTDP-BTMPP- and CHCA-derived Positive Ions and their IYs in CHCA-MALDI of THTDP-BTMPP (Further Abbreviated as T-B) Measured as a Function of the Analyte Amount in $25 \mathrm{nmol} \mathrm{CHCA}$

\begin{tabular}{lcclll}
\hline \multirow{2}{*}{ T-B, pmol } & \multicolumn{2}{c}{ Ions from sample } & & \multicolumn{2}{c}{ IY } \\
\cline { 2 - 3 } \cline { 5 - 6 } & T-B, $10^{8}$ & CHCA, $10^{9}$ & & T-B, $10^{-4}$ & CHCA, 10 \\
& & & & & \\
\hline 0.1 & $0.45 \pm 0.07$ & $1.5 \pm 0.4$ & & $7.4 \pm 1.2$ & $1.0 \pm 0.3$ \\
0.3 & $1.1 \pm 0.4$ & $1.7 \pm 0.4$ & & $6.1 \pm 2.2$ & $1.1 \pm 0.3$ \\
1 & $3.7 \pm 1.4$ & $1.6 \pm 0.2$ & & $6.2 \pm 2.3$ & $1.0 \pm 0.1$ \\
3 & $7.7 \pm 0.9$ & $1.3 \pm 0.1$ & & $4.3 \pm 0.5$ & $0.88 \pm 0.07$ \\
10 & $11 \pm 2$ & $1.2 \pm 0.2$ & & $1.7 \pm 0.3$ & $0.78 \pm 0.09$ \\
30 & $15 \pm 1$ & $0.69 \pm 0.10$ & & $0.81 \pm 0.05$ & $0.46 \pm 0.07$ \\
100 & $22 \pm 5$ & $0.49 \pm 0.08$ & & $0.36 \pm 0.07$ & $0.33 \pm 0.05$ \\
\hline
\end{tabular}

the melting point decreased. One may argue that a CHCAderived anion such as $[\mathrm{CHCA}-\mathrm{H}]^{-}$acts as the common counter-ion for $\mathrm{BTPP}^{+}, \mathrm{BMI}^{+}$, and $\mathrm{THTDP}^{+}$and that the intercharge distances and the Coulombic potentials in the three ion pairs are rather similar. However, based on the sensitivity of the calculated equilibrium constant to the Coulombic potential, we are reluctant to accept the explanation until further data are available. We would rather interpret the above results as an indication that the equilibrium thermodynamic treatment of the neutralization reaction may be inappropriate for MALDI of salts.

In a recent computational study of MALDI of a matrix, 2,5-dihydroxybenzoic acid (DHB), Knochenmuss and Zhigilei [26] suggested that MALDI was a race between recombination and separation of charges. Compared WITH Langevin-type reactions, a recombination via tunneling in the Marcus inverted region could occur slowly, which was suggested as a major reason why MALDI was viable as an analytical method. Once verified experimentally, the tunneling model must be taken into account in an effort to find an explanation for the very small IY for CHCA, around $10^{-7}$.

\section{Concentration Dependence of IY}

According to the data in Tables 1, 2 and 3, the number of analyte ions from a sample increased with their concentration in the solid sample. In the case of the CHCA-derived ions, their number remained nearly constant at low analyte concentration, but decreased at higher concentration. Rather than the number of ions formed, IY may be a better measure in the study of the concentration dependence. IY data for BTPP-Cl in Table 1 are drawn as a function of its concentration in Figure 4 for visual inspection (those for the samples containing $\mathrm{BMI}^{-\mathrm{PF}_{6}}$ and THTDP-BTMPP display similar patterns, as shown in the Supplementary Material).

A noticeable feature in Figure 4 is that IYs for BTPP-Cl and CHCA, which were nearly constant at low analyte concentration, decreased steadily as the analyte concentration increased. In the case of MALDI of peptides, the decrease of the matrix-derived signals, called the 'matrix suppression effect $[7,8]$ ', is often taken as evidence for the gas-phase proton transfer model. That is, as the concentration of a neutral peptide increases in the plume, the
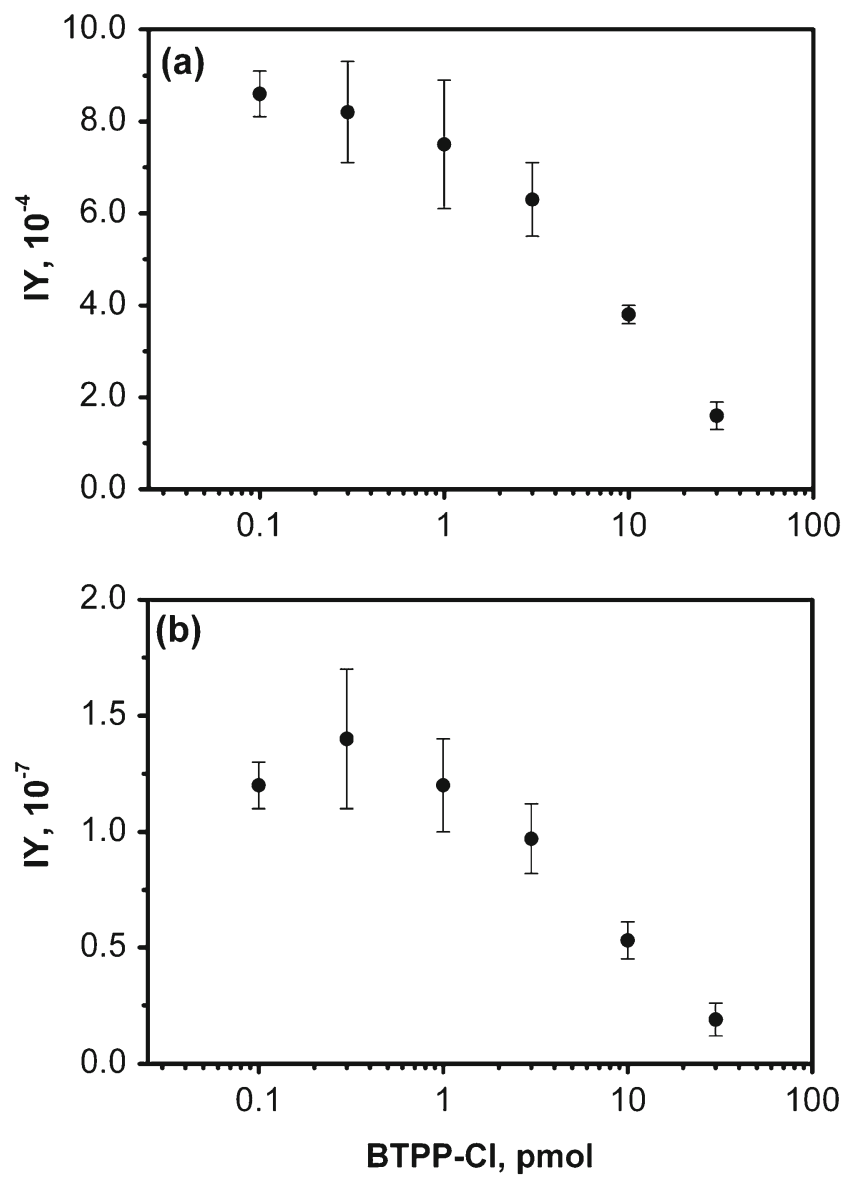

Figure 4. IYs for (a) BTPP-Cl and (b) CHCA versus the amount of BTPP-Cl in solid samples containing $25 \mathrm{nmol}$ CHCA. $4.5 \mu \mathrm{J}$ per pulse of laser energy was used

equilibrium for the proton transfer reaction such as $\mathrm{P}+\mathrm{MH}^{+}$ $\rightarrow \mathrm{PH}^{+}+\mathrm{M}$ shifts toward the right and, hence, suppresses the intensities of matrix-derived ions such as $\mathrm{MH}^{+}$. For the time being, let us assume that proton transfer is not involved in the formation of gas-phase $\mathrm{BTPP}^{+}$. Then, the matrix suppression effect observed in this work must occur through neutral ionpair formation, which becomes more prevalent at higher analyte concentration. The ion-pair formation will dominate the charge separation wherever it may occur, whether in the selvedge or in the gas phase, or when the solvent evaporates during sample preparation.

Another interesting aspect of the present data is that the decrease of IY becomes observable at around 3 pmol of BTPP-Cl. At this concentration, the numbers of BTPP-Cland CHCA-derived cations are comparable, $1.1 \times 10^{9}$ versus $1.4 \times 10^{9}$. Let us assume that the same numbers of positive and negative ions are formed from BTPP-Cl, and also from CHCA. Then the above observation can be explained as follows. At very low concentration of BTPP-Cl, it is mostly the CHCA-derived negative ions that act as the counter ions for $\mathrm{BTPP}^{+}$and for the CHCA-derived positive ions. Since the number of such counter anions remains nearly constant, IYs for BTPP-Cl and CHCA also remain constant. As the 
concentration of BTPP-Cl increases, the number of $\mathrm{Cl}^{-}$, which also acts as a counter anion, increases. As the extent of neutralization by $\mathrm{Cl}^{-}$gets comparable to or larger than by CHCA-related anions, the suppression of both analyte- and matrix-derived cation intensities becomes significant and hence visible.

\section{Generation of Gas-Phase Cations}

As the first step in our effort to find the reason for smaller than expected IY for salts, we checked whether BTPP-Cl was completely depleted from the target at all. In the intensity versus time patterns (Figure 1), the analyte signal tended to deplete a little more slowly than the matrix signal. Suspecting that some analyte might remain on the target even after the complete depletion of the matrix, we depleted an extended area on the target, added the matrix solution to dissolve the remaining analyte, dried it, and attempted MALDI again. Hardly any analyte signal was observed. That is, IY for BTPP-Cl was small not because most of it remained on the target. This suggested that most of BTPP-Cl was depleted as neutral forms such as the neutral ion pair of $\mathrm{BTPP}^{+}-\mathrm{Cl}^{-}$.

The formation of ion pairs will be effective not only in the gas phase but also in the solid sample - an ionic species in the solid sample will be called a preformed ion whether the ion is part of an ion pair or not. Assuming that the cation and the anion of an ion pair in a solid sample are not easily released as free gas-phase ions, we propose two models to explain very small IY values for salts. In Model 1, we postulate that the gas-phase cations released by MALDI originate from free cations in the solid sample, which constitute a tiny fraction of preformed cations. In Model 2, preformed cations are initially released in the form of neutral ion pairs. Then, the ion pairs lose the anions via reaction with matrix-derived cations such as shown below.

$$
\mathrm{BTPP}^{+} \mathrm{Cl}^{-}+\mathrm{MH}^{+} \rightarrow \mathrm{BTPP}^{+}+\mathrm{M}+\mathrm{HCl}
$$

Also to be noted is that both of the models are compatible with the concentration effect.

The basic thermodynamics of gas-phase ion formation from a charged species in solid phase was discussed by Zenobi and Knochenmuss [4]. They noted that the separation of $\mathrm{Na}^{+}$or $\mathrm{Cl}^{-}$in a $\mathrm{NaCl}$ crystal needs $810 \mathrm{~kJ} \mathrm{~mol}^{-1}$ or $8.4 \mathrm{eV}$ and that the energy requirement can be met by the absorption of three photons at $337 \mathrm{~nm}$. In the photoexcitation of large polyatomic ions, absorbed photon energy is converted to the internal energy, which is mostly vibrational. According to our approximation presented above, the binding energy of $810 \mathrm{~kJ} \mathrm{~mol}^{-1}$ results in $K_{\text {neutral }} \approx 2 \times 10^{42}$ at $1000 \mathrm{~K}$. That is, under a typical MALDI condition, the emission of a polyatomic ion (charge separation) is completely dominated by the reverse process (neutralization). Zenobi and Knochenmuss proposed that the dielectric screening by residual solvent, matrix, or non-ionic parts of large molecules would reduce the Coulombic potential for an ion and hence allow easy release of such an ion from the solid to the gas phase. Based on the argument by Zenobi and Knochenmuss, our Model 1 can be further elaborated as follows. When the solvent is evaporated, most of the cations of a salt will form ion pairs. Such ion pairs will be released as neutrals upon ablation. However, since the number of matrix neutral molecules is overwhelmingly larger than that of ions in the solid sample, there will be a small number of dielectrically screened ions, which can be readily released into the gas phase and contribute to small IYs observed. Similar IYs for the three salts studied means that the number of dielectrically screened ions is not determined by the neutralization equilibrium, but by the ion-to-matrix neutral ratio and, hence, is similar at the same concentrations of different salts. In this regard, Model 1 may be called the 'dielectrically screened preformed ion emission' model.

Based on the Spengler's [27] and our own [28, 29] studies on MALDI plumes, let us take $10 \mathrm{~atm}$ and $1000 \mathrm{~K}$ as the typical pressure and temperature, respectively, during the ablation and in the early plume. Under this condition, the collision frequency for a molecule with a relative molecular mass of 300 and a hard sphere diameter of $10 \AA$ is $8.6 \times 10^{10} \mathrm{~s}^{-1}$. According to the pressure-distance curve reported by Spengler [27], most of the collisions in MALDI may occur during the first $10 \mathrm{~ns}$, resulting in roughly $10^{3}$ collisions. In the present work, one out of $10^{7} \mathrm{CHCA}$ molecules in the plume was found to be in the forms of positively or negatively charged ions. This roughly means that a neutral ion pair containing $\mathrm{BTPP}^{+}$ion will encounter a matrix-derived cation once in every $10^{7}$ collisions. That is, the probability for the ion pair to encounter a cation will be around $10^{-4}\left(=10^{3} \times 10^{-7}\right)$. The estimate of IY for analytes based on Model 2 is rather close to the experimental data for salts $\left(<10^{-3}\right)$.

Discussion made so far suggests that Models 1 and 2 are compatible with the present experimental data and observations. In Model 1, dielectrically screened preformed ions are emitted into the gas phase by MALDI. In Model 2, preformed ions are emitted in the form of ion pairs and the anion loss from the ion pairs is aided by matrix-derived cations.

\section{Conclusion}

In a solid matrix-salt mixture, cations and anions of the salt are present in the form of preformed ions. Intuitively, such preformed ions might be easily released into the gas phase by laser-induced ablation. Against such a naive expectation, IYs measured for some salts were small, less than $10^{-3}$. IY for the matrix (CHCA), around $10^{-7}$, was also smaller than previous measurements and speculation. Two models have been proposed to explain the very small IYs for salts and the concentration effects, both of which are based on the fact that the cation-anion neutralization equilibrium is highly shifted toward the neutral ion pair. In Model 1, gas-phase 
cations of salts originate from a small fraction of preformed cations in the solid sample, which are screened from counter anions via solvation. In Model 2, gas-phase cations of the salts are formed by a reaction between a neutral ion pair and matrix-derived cations.

Investigation in this work was limited to salts, with the hope that the presence of preformed ions in solid matrix-salt mixtures would allow only one mechanism for ion formation, i.e., preformed ion emission. However, the final outcome turned out to be more complicated. In particular, participation of gas-phase ion-molecule reactions could not be ruled out. Regardless, present results would be a useful benchmark in the mechanistic study of peptide ion formation, which is being planned.

\section{Acknowledgments}

The authors acknowledge financial support for this work by the National Research Foundation, Republic of Korea. Y.S. S. and Y.J.B. thank the Ministry of Education, Science, and Technology, Republic of Korea, for Brain Korea 21 Fellowship.

\section{References}

1. Hillenkamp, F., Peter-Katalinić, J.: MALDI MS. A Practical Guide to Instrumentation, Methods and Applications. Wiley-VCH, Weinheim (2007)

2. Cole, R.B.: Electrospray and MALDI Mass Spectrometry. Fundamentals, Instrumentation, Practicalities, and Biological Applications, 2nd edn. Wiley, Hoboken (2010)

3. Dreisewerd, K.: The desorption process in MALDI. Chem. Rev. 103, 395-425 (2003)

4. Zenobi, R., Knochenmuss, R.: Ion formation in MALDI mass spectrometry. Mass Spectrom. Rev. 17, 337-366 (1998)

5. Knochenmuss, R., Stortelder, A., Breuker, K., Zenobi, R.: Secondary ion-molecule reactions in matrix-assisted laser desorption/ionization. $J$. Mass Spectrom. 35, 1237-1245 (2000)

6. Breuker, K., Knochenmuss, R., Zhang, J., Storteler, A., Zenobi, R.: Thermodynamic control of final ion distributions in MALDI: In-plume proton transfer reactions. Int. J. Mass Spectrom. 226, 211-222 (2003)

7. Knochenmuss, R., Zenobi, R.: MALDI ionization: The role of in-plume processes. Chem. Rev. 103, 441-452 (2003)

8. Knochenmuss, R.: Ion formation mechanisms in UV-MALDI. Analyst 131, 966-986 (2006)

9. Karas, M., Glückmann, M., Schäfer, J.: Ionization in matrix-assisted laser desorption/ionization: Singly charged molecular ions are the lucky survivors. J. Mass Spectrom. 35, 1-12 (2000)

10. Karas, M., Krüger, R.: Ion formation in MALDI: The cluster ionization mechanism. Chem. Rev. 103, 427-439 (2003)

11. Jaskolla, T.W., Karas, M.: Compelling evidence for lucky survivor and gas phase protonation: The unified MALDI analyte protonation mechanism. J. Am. Soc. Mass Spectrom. 22(6), 976-988 (2011)
12. Ens, W., Mao, Y., Mayer, F., Standing, K.G.: Properties of matrixassisted laser desorption. Measurements with a time-to-digital converter. Rapid Commun. Mass Spectrom. 5(3), 117-123 (1991)

13. Mowry, C.D., Johnston, M.V.: Simultaneous detection of ions and neutrals produced by matrix-assisted laser desorption. Rapid Commun. Mass Spectrom. 7, 569-575 (1993)

14. Quist, A.P., Huth-Fehre, T., Sundqvist, B.U.R.: Total yield measurements in matrix-assisted laser desorption using a quartz crystal microbalance. Rapid Commun. Mass Spectrom. 8, 149-154 (1994)

15. Puretzky, A.A., Geohegan, D.B.: Gas-phase diagnostics and LIFimaging of 3-hydroxypicolinic acid MALDI-matrix plumes. Chem. Phys. Lett. 286, 425-432 (1998)

16. Yoon, S.H., Kim, M.S.: Development of a time-resolved method for photodissociation mechanistic study of protonated peptides: Use of a voltage-floated cell in a tandem time-of-flight mass spectrometer. J. Am. Soc. Mass Spectrom. 18, 1729-1739 (2007)

17. Bae, Y.J., Yoon, S.H., Moon, J.H., Kim, M.S.: Optimization of reflectron for kinetic and mechanistic studies with multiplexed multiple tandem $\left(\mathrm{MS}^{n}\right)$ time-of-flight mass spectrometry. Bull. Korean Chem. Soc. 31(1), 92-99 (2010)

18. Yoon, S.H., Moon, J.H., Kim, M.S.: Changes in dissociation efficiency and kinetics of peptide ions induced by basic residues and their mechanistic implication. J. Am. Soc. Mass Spectrom. 22, 214-220 (2011)

19. Moon, J.H., Yoon, S.H., Kim, M.S.: Temperature of peptide ions generated by matrix-assisted laser desorption ionization and their kinetic parameters. J. Phys. Chem. B 113, 2071-2076 (2009)

20. Beavis, R.C., Chait, B.T.: Factors affecting the ultraviolet laser desorption of proteins. Rapid Commun. Mass Spectrom. 3(7), 233237 (1989)

21. Westman, A., Brinkmalm, G., Barofsky, D.F.: MALDI induced saturation effects in chevron microchannel plate detectors. Int. J. Mass Spectrom. Ion Processes 169/170, 79-87 (1997)

22. Frankevich, V., Knochenmuss, R., Zenobi, R.: The origin of electrons in MALDI and their use for sympathetic cooling of negative ions in FTICR. Int. J. Mass Spectrom. 220, 11-19 (2002)

23. Knochenmuss, R.: Photoionization pathways and free electrons in UVMALDI. Anal. Chem. 76, 3179-3184 (2004)

24. McCombie, G., Knochenmuss, R.: Enhanced MALDI ionization efficiency at the metal-matrix interface: Practical and mechanistic consequences of sample thickness and preparation method. J. Am. Soc. Mass Spectrom. 17, 737-745 (2006)

25. Armstrong, D.W., Zhang, L., He, L., Gross, M.L.: Ionic liquids as matrixes for matrix-assisted laser desorption/ionization mass spectrometry. Anal. Chem. 73, 3679-3686 (2001)

26. Knochenmuss, R., Zhigilei, L.V.: What determines MALDI ion yields? A molecular dynamics study of ion mechanisms. Anal. Bioanal. Chem (2011). doi:10.1007/s00216-011-5194-x

27. Spengler, B.: Post-source decay analysis in matrix-assisted laser desorption/ionization mass spectrometry of biomolecules. J. Mass Spectrom. 32, 1019-1036 (1997)

28. Yoon, S.H., Moon, J.H., Kim, M.S.: A comparative study of in- and post-source decays of peptides and preformed ions in matrix-assisted laser desorption ionization time-of-flight mass spectrometry: Effective temperature and matrix effect. J. Am. Soc. Mass Spectrom. 21, 18761883 (2010)

29. Bae, Y.J., Moon, J.H., Kim, M.S.: Expansion cooling in the matrix plume is under-recognized in MALDI mass spectrometry. J. Am. Soc. Mass Spectrom. 22, 1070-1078 (2011) 